\title{
Anisotropy of the proton kinetic energy in ice Ih
}

\author{
Y. Finkelstein,,$^{*}$ R. Moreh, ${ }^{2}$ F. Bianchini, ${ }^{3}$ and P. Vajeeston ${ }^{3}$ \\ ${ }^{1}$ Chemistry Division, Nuclear Research Center Negev (NRCN), Beer-Sheva 84190, Israel \\ ${ }^{2}$ Physics Department, Ben-Gurion University of the Negev, Beer-Sheva 84105, Israel \\ ${ }^{3}$ Center for Materials Sciences and Nanotechnology, Department of Chemistry, University of Oslo, N-0315 \\ Oslo, Norway
}

\begin{abstract}
The partial vibrational density of states ( $p$ VDOS) of ice Ih, as simulated by first principle modeling based on density functional theory (DFT), is utilized for computing the Cartesian components of the proton and oxygen quantum kinetic energies, $\operatorname{Ke}(\mathrm{H})$ and $\operatorname{Ke}(\mathrm{O})$ respectively, along and perpendicular to the hydrogen bonds. The DFT method was found to yield better agreement with deep inelastic neutron scattering (DINS) measurements than the semi empirical (SE) calculations. The advantage of using the DFT method is to enable us to resolve the external and internal phonon bands of the Cartesian projections of the $p$ VDOS, and hence those of the lattice and vibrational components of $\mathrm{Ke}(\mathrm{H})$. We show that a $p$ VDOS analysis is a valuable tool in testing scattering results of complex systems and suggest its potential to explore competing quantum effects, e.g. on $\operatorname{Ke}(\mathrm{H})$ across phase transitions in water.
\end{abstract}

\section{Introduction}

A substantial body of experimental work exists on the nuclear nuclear kinetic energy of the proton, $\mathrm{Ke}(\mathrm{H})$, in ice and water. Only one scattering technique, namely deep inelastic neutron scattering (DINS), was utilized for measuring $\mathrm{Ke}(\mathrm{H})$ in $\mathrm{H}_{2} \mathrm{O}$ phases. Out of those, DINS is regarded as the only standard method for direct $\mathrm{Ke}(\mathrm{H})$ determinations of the proton zero point kinetic energy (ZPKE), via a measure of the proton radial momentum distribution (RMD). Note that the kinetic energy of the $\mathrm{H}$-atom in $\mathrm{H}_{2} \mathrm{O}$ up to $\sim 280 \mathrm{~K}$ is practically equal to its ZPKE. ${ }^{1}$ Currently, measuring the ZPKE is provided only by the Vesuvio spectrometer at ISIS (Rutherford Appleton Laboratory, UK). Worth mentioning in that regard, is that $\mathrm{Ke}(\mathrm{H})$ may also be determined by inelastic neutron scattering (INS). ${ }^{2}$ Significantly different from DINS however, in INS one measures the fundamental frequencies of the condensed 
$\mathrm{H}_{2} \mathrm{O}$ phase. These INS data are normally used as input for calculating $\mathrm{Ke}(\mathrm{H})$ in the semi empirical (SE) method in which the harmonic approximation (HA) is assumed. ${ }^{3}$

Lately, an extensive experimental and theoretical emphasis is given in the literature for measuring and computing the Cartesian components of $\mathrm{Ke}(\mathrm{H})$ along and perpendicular to the hydrogen bond ( $\mathrm{HB}$ ) direction in $\mathrm{H}_{2} \mathrm{O} .^{2,4,5,6,7,8,9}$ These components and were found to provide a direct estimate of competing quantum effects (CQEs). ${ }^{5}$ By now, such components in light water in the form of ice Ih were measured directly only by DINS ${ }^{2,8}$ and calculated semi empirically by utilizing INS measurements. ${ }^{2}$

In the present work we report on the first DFT simulation of the Cartesian components of the $\mathrm{H}-\mathrm{VDOS}$ in ice Ih from which the directional components of $\mathrm{Ke}(\mathrm{H})$ along and perpendicular to the hydrogen bond are resolved. It is encouraging that the currently deduced values are in good agreement with available measured DINS data on solid $\mathrm{H}_{2} \mathrm{O}$, a result which emphasizes the validity of the $\mathrm{pVDOS}$ approach as a powerful tool in exploring proton dynamics of $\mathrm{HB}$ containing systems.

\section{Theoretical Remarks}

In the following we emphasize the advantages of using a $p$ VDOS analysis over the SE approach for treating the directional $\mathrm{Ke}(\mathrm{H})$ in ice.

\section{pVDOS vs SE}

The SE approach. ${ }^{2,3,1}$ is used to compute $\mathrm{Ke}(\mathrm{H})$ in condensed $\mathrm{H}_{2} \mathrm{O}$ phases where it is assumed that the coupling of the $\mathrm{H}_{2} \mathrm{O}$ modes of motion to the HBs network is fully accounted for by utilizing the measured frequencies of $\mathrm{H}_{2} \mathrm{O}$ and its geometric parameters $\left(\mathrm{R}_{\mathrm{OH}}\right.$ and the $\mathrm{HOH}$ angle). For $\mathrm{H}_{2} \mathrm{O}$, five discrete frequencies exist arising from the two lattice modes (translation and libration of the entire $\mathrm{H}_{2} \mathrm{O}$ molecule) and from its three internal vibrations (OHO bending and two $\mathrm{OH}$ stretching modes). Theses frequencies are then reconstructed by solving the equations of motion of the oxygen atom and two protons of $\mathrm{H}_{2} \mathrm{O}$. This procedure yields the atomic amplitudes along any direction, thus providing the energy fractions shared by the constituent atom in each mode of motion along any direction. These fractions are used to deduce the Cartesian XYZ components of $\mathrm{Ke}(\mathrm{H})$ and $\mathrm{Ke}(\mathrm{O})$.

The SE approach was found to work flawlessly as it successfully predicted DINS $\operatorname{Ke}(\mathrm{H}, \mathrm{D}, \mathrm{O})$ values in various phases of $\mathrm{H}(\mathrm{D})_{2}$ and $\mathrm{H}(\mathrm{D})_{2} \mathrm{O} .{ }^{10,11}$ In fact, it was established as a powerful 
tool for predicting atomic KE values (already way back in the $70^{\text {th }} / 90^{\text {th }}$ ) of ${ }^{15} \mathrm{~N} /{ }^{13} \mathrm{C}$, contained in a variety of molecular systems; the results were in excellent agreement with nuclear resonance photon scattering/fluorescence (NRPS/NRF) experiments. ${ }^{12,13,14,15,16,17}$

It is also very interesting to note that despite the fact that the SE method relies on the HA, it was found to be applicable to cases involving strong anharmonic potentials. ${ }^{18}$ Examples include: ${ }^{18}$ ferroelectric crystals of the $\mathrm{KDP}$ family $\left(\mathrm{MH}_{2} \mathrm{PO}_{4} ; \mathrm{M}=\mathrm{K}, \mathrm{Rb}, \mathrm{Cs}\right)$, and the super protonic conductor $\mathrm{Rb}_{3} \mathrm{H}\left(\mathrm{SO}_{4}\right)_{2}$ in which $\mathrm{HB}$ symmetrization is very nearly realized. In such systems, it is necessary to account for the pVDOS, i.e. the part of the VDOS shared by the protons (H-VDOS), as simulated by DFT or molecular dynamics (MD). ${ }^{18}$ Note that the $p$ VDOS approach is more accurate than the SE method as it accounts for both the continuous phonon distribution of the system and for the fraction shared by the proton in all vibrational states (phonons). Moreover, the energy fractions distribution is self-contained in the $p$ VDOS and it is unnecessary to calculate it in advance as in the SE approach.

A breakdown of the SE approach in predicting $\mathrm{Ke}(\mathrm{H})$ values was first demonstrated in DINS studies of nanoconfined $\mathrm{H}_{2} \mathrm{O}$ where anomalous $\mathrm{Ke}(\mathrm{H})$ deviations of $\pm 30-50 \%$ (from the standard value of $\mathrm{Ke}(\mathrm{H}) \sim 152 \mathrm{meV}$ measured in e.g. ice) were reported. These were found in cases such as protein hydration shell water ${ }^{19}$ and in water nanoconfined in silica gels, ${ }^{20,21}$ in carbon nanotubes ${ }^{22}$ and in Beryl. ${ }^{23}$ These anomalies couldn't be captured however by the SE approach nor by more sophisticated theoretical calculations such as path integral molecular dynamics (PIMD) $)^{24,25,26}$ and path integral monte Carlo (PIMC) 27,28,29,26 calculations. Only very recently, a first success was reported by utilizing first principle based DFT simulations of the partial $\mathrm{H}-\mathrm{VDOS}$ in the $\mathrm{H}_{2} \mathrm{O} @$ Berryl system. ${ }^{30}$ By treating the whole system, of the hosting Beryl lattice and the confined $\mathrm{H}_{2} \mathrm{O}$, as a single quantum system, the deduced $\mathrm{Ke}(\mathrm{H})$ at $5 \mathrm{~K}$ was found to conform precisely to the DINS measured anomalous value, $\mathrm{Ke}(\mathrm{H})=104 \mathrm{meV} .{ }^{23}$ The key insight here is that under nano-confinement, the coupling of the water molecule to host lattice modes yield proton states distinctly different from those of ordinary water phases. ${ }^{31}$ This success was in fact the first accurate theoretical reconstruction of an anomalous DINS $\mathrm{Ke}(\mathrm{H})$ value in nanoconfined water, by which the crucial importance of the coupling of the water modes of motion to host lattice modes in calculating KEs was realized .

\section{SE calculation}


In the following we stick to the notation of Ref. ${ }^{2}$ by selecting the direction of $\mathrm{OH} 1$ covalent bond to be the same as the $\mathrm{Z}$ Cartesian direction and along the HB direction in ice Ih. The relative directions of the abc crystallographic axes and the Cartesian XYZ ones are defined in Fig. 1.

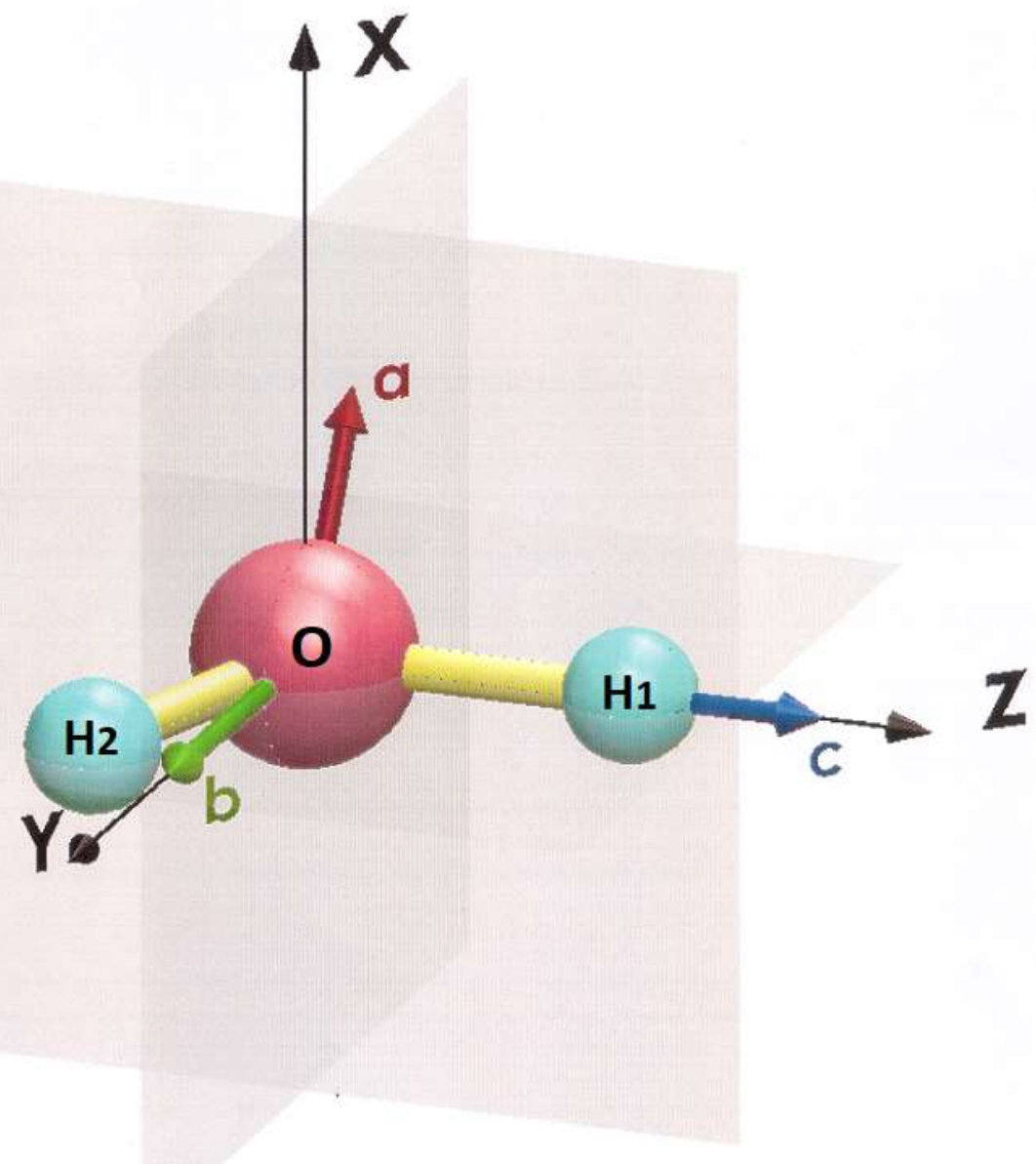

Fig. 1 Definition of the $\mathrm{XYZ}$ Cartesian directions in $\mathrm{H}_{2} \mathrm{O}$ : the $\mathrm{OH}_{1}$ covalent bond is along $\mathrm{z}$, and the $(\mathrm{Y}, \mathrm{Z})$ plane coincides with the $\mathrm{H}_{2} \mathrm{O}$ molecular plane. The relation of the Cartesian axes frame to the abc crystallographic one of hexagonal ice (see Fig. 2), is also shown: $c \| Z$, $(\mathrm{b}, \mathrm{c}) \|(\mathrm{Y}, \mathrm{Z}), \mathrm{b} \perp \mathrm{c}, \mathrm{a} \perp \mathrm{c}, \angle \mathrm{ab}=120^{\circ}\left(\angle \mathrm{aX}=30^{\circ}, \angle \mathrm{Yb} \sim 17^{\circ}\right)$.

Accounting for the directions defined in Fig. 1, the following relations apply to the $\mathrm{H}_{1}$ atom: ${ }^{2,32}$

$\operatorname{Ke}_{\mathrm{x}}\left(\mathrm{H}_{1}\right)=\mathrm{S}_{\mathrm{t}} \alpha\left(\mathrm{U}_{\mathrm{t}}\right)+\left(\mathrm{S}_{\mathrm{Ry}}+\mathrm{S}_{\mathrm{Rz}}\right) \alpha\left(\mathrm{U}_{\mathrm{R}}\right)$
$\mathrm{Ke}_{\mathrm{y}}\left(\mathrm{H}_{1}\right)=\mathrm{S}_{\mathrm{t}} \alpha\left(\mathrm{U}_{\mathrm{t}}\right)+\mathrm{S}_{\mathrm{Rx}} \alpha\left(\mathrm{U}_{\mathrm{R}}\right)+\mathrm{S}_{2} \alpha\left(\mathrm{U}_{2}\right)$ 
$\mathrm{Ke}_{\mathrm{z}}\left(\mathrm{H}_{1}\right)=\mathrm{S}_{\mathrm{t}} \alpha\left(\mathrm{v}_{\mathrm{t}}\right)+\mathrm{S}_{1} \alpha\left(\mathrm{v}_{1}\right)+\mathrm{S}_{3} \alpha\left(\mathrm{v}_{3}\right)$

$\mathrm{S}_{\mathrm{t}}, \mathrm{S}_{\mathrm{Rx}, \mathrm{y}, \mathrm{z}}, \mathrm{S}_{1,2,3}$ are the $\mathrm{KE}$ fractions shared by $\mathrm{H}_{1}$ in the translational, rotational (around the Cartesian axes passing through the molecular center of mass) and the three internal frequencies of $\mathrm{H}_{2} \mathrm{O}$ respectively. $\alpha\left(\mathrm{v}_{j}\right)=\frac{h v_{j}}{2}\left(\frac{1}{e \frac{h v_{j}}{\mathrm{kT}}-1}+\frac{1}{2}\right)$, is the KE of the corresponding Planck oscillator, with $v_{j}(j=\mathrm{t}, \mathrm{r}, \mathrm{bnd}, \mathrm{ss}$, as $)$ the measured characteristic frequency of translation, libration and three internal (bending and symmetric and asymmetric stretch) modes of motion of $\mathrm{H}_{2} \mathrm{O}$. It should be noted that in this simplified model, one accounts in each mode for the same characteristic frequency (measured for the studied water phase) regardless of the direction, thus coupling effects of the water molecule to surrounding lattice modes are ignored.

\section{$p$ VDOS calculation}

A detailed discussion of the KE calculation by the $p$ VDOS method may be found elsewhere. ${ }^{18}$ Here we just mention that by accounting for the $l^{\text {th }}$ Cartesian component $(l=\mathrm{X}, \mathrm{Y}, \mathrm{Z})$ of the H-VDOS, $g_{H_{l}}(v)$, the directional component of $\mathrm{Ke}(\mathrm{H}), K e_{l}(H)$, may be deduced directly by:

$$
K e_{l}(H)=\frac{\frac{3}{2} \int_{v_{0}}^{v_{f}} g_{H_{l}}(v) \alpha(v, T) d v}{\int_{v_{0}}^{v_{f}} g_{H_{l}}(v) d v}
$$

with $v_{0}$ and $v_{f}$ the frequency limits of $g_{H_{l}}(v)$.

\section{DFT simulation of $g_{H_{l}}(\mathrm{v})$}

Total energies have been calculated using the projected-augmented plane-wave (PAW) implementation of the Vienna $a b$ initio simulation package (VASP). ${ }^{33,34}$ All these calculations were made with the Perdew, Burke, and Ernzerhof (PBE) $)^{35}$ exchange correlation functional. Ground-state geometries were determined by minimizing stresses and HellmanFeynman forces using the conjugate-gradient algorithm with a force convergence threshold of $10^{-3} \mathrm{eV} \AA^{-1}$. Brillouin zone integration was performed with a Gaussian broadening of 0.1 $\mathrm{eV}$. From various sets of calculations it was found that $512 \mathbf{k}$ points in the Brillouin zone and a $600 \mathrm{eV}$ plane-wave cut-off are sufficient to ensure optimum accuracy in the computed 
results. The k-points were generated using the Monkhorst-Pack method with a grid size of $8 \times 8 \times 8$.

A frozen phonon calculation was performed on suitably large supercells using the Phonopy program to obtain the phonon dispersion curve and density of states. ${ }^{36}$ An atomic displacement of $0.0075 \AA$ was used with a symmetry consideration to obtain the force constants for the phonon calculations. The displacements in opposite directions along all possible axes were included in the calculations to improve the overall accuracy. The forces are calculated using the VASP code and the resulting data were imported into the Phonopy program. The dynamical matrices were calculated from the force constants, and the phonon DOS (PDOS) curves were computed using the Monkhorst-Pack scheme. ${ }^{37}$ Hexagonal ice (ice Ih) is in Space group P63/mmc, 194; analogous to $\beta$-tridymite silica or lonsdaleite, having a six-fold screw axis (rotation around an axis in addition to a translation along the axis). ${ }^{38}$ It possesses a fairly open low-density structure, where the packing efficiency is low $(\sim 1 / 3)$ compared with simple cubic $(\sim 1 / 2)$ or face-centered cubic $(\sim 3 / 4)$ structures. In this atomic arrangement, all molecules experience identical molecular environments. The directional dependent PDOS where computed along (001), (010) and (100). ${ }^{39}$

The simulated structure of hexagonal ice is presented in Fig. 2.
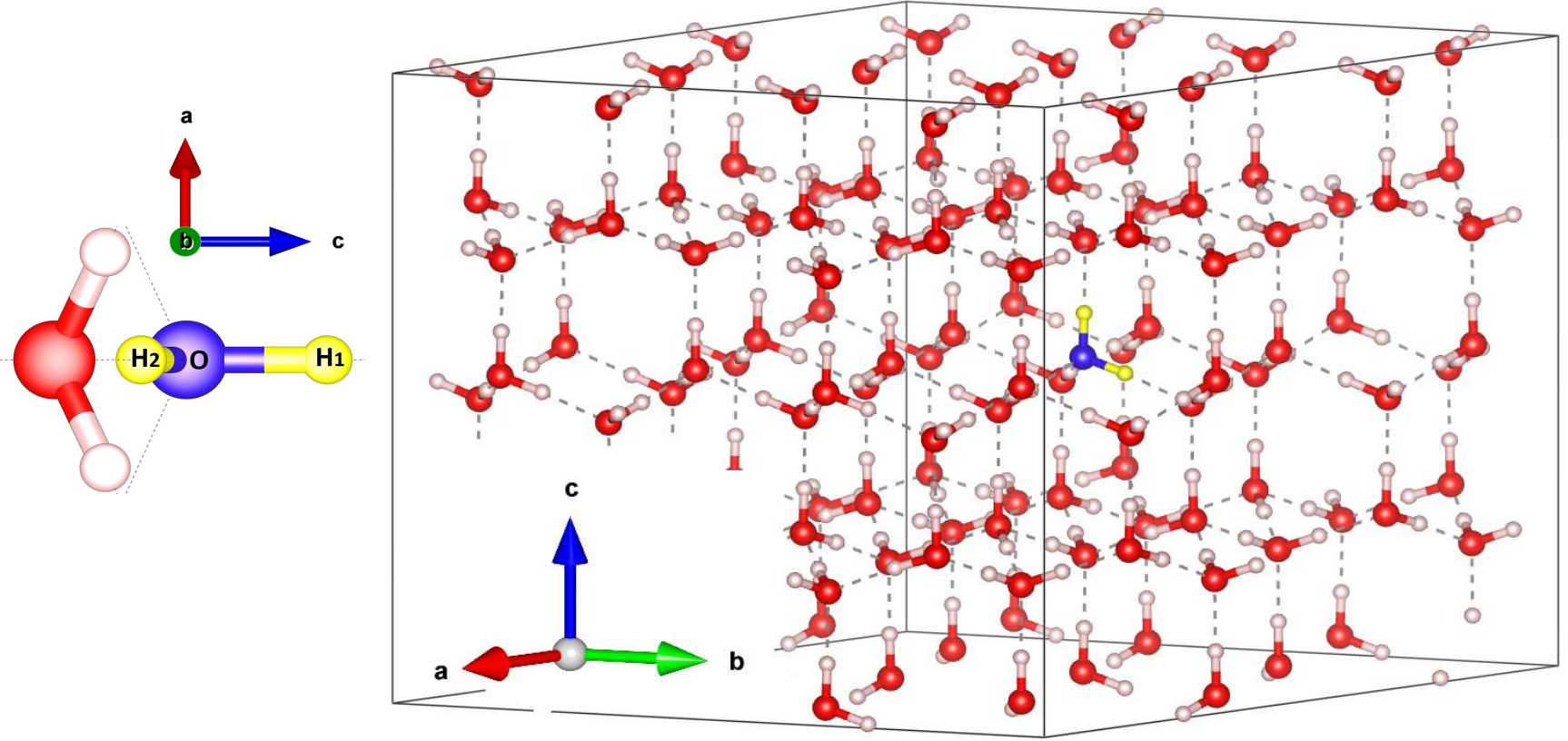
Fig. 2 Simulated structure of ice Ih (O-red, H-white). The blue/yellow colored $\mathrm{H}_{2} \mathrm{O}$ represents a crystalline molecule which is oriented relative to the crystallographic axes as in the left inset: $\mathrm{OH}_{1}$ bond $\| \mathrm{c}, \mathrm{H}_{1} \mathrm{OH}_{2}$ molecular plane parallel to the (b,c) plane, a $\perp$ molecular plane (shown for clarity in the left inset for a b direction view). The current $\mathrm{Ke}(\mathrm{H})$ analysis refers to the Cartesian components of the $\mathrm{H}_{1}$-VDOS according to the directions of Fig. 1.

\section{Results and Discussion}

Fig. 3 depicts the calculated Cartesian components of the $\mathrm{H}_{1}$-VDOS in ice Ih. Fig. 4 compares the directional partial VDOS of the two protons in $\mathrm{H}_{2} \mathrm{O}$. In the two figures the Cartesian directions correspond to $\mathrm{H}_{2} \mathrm{O}$ molecular orientation as defined in Fig. 1.

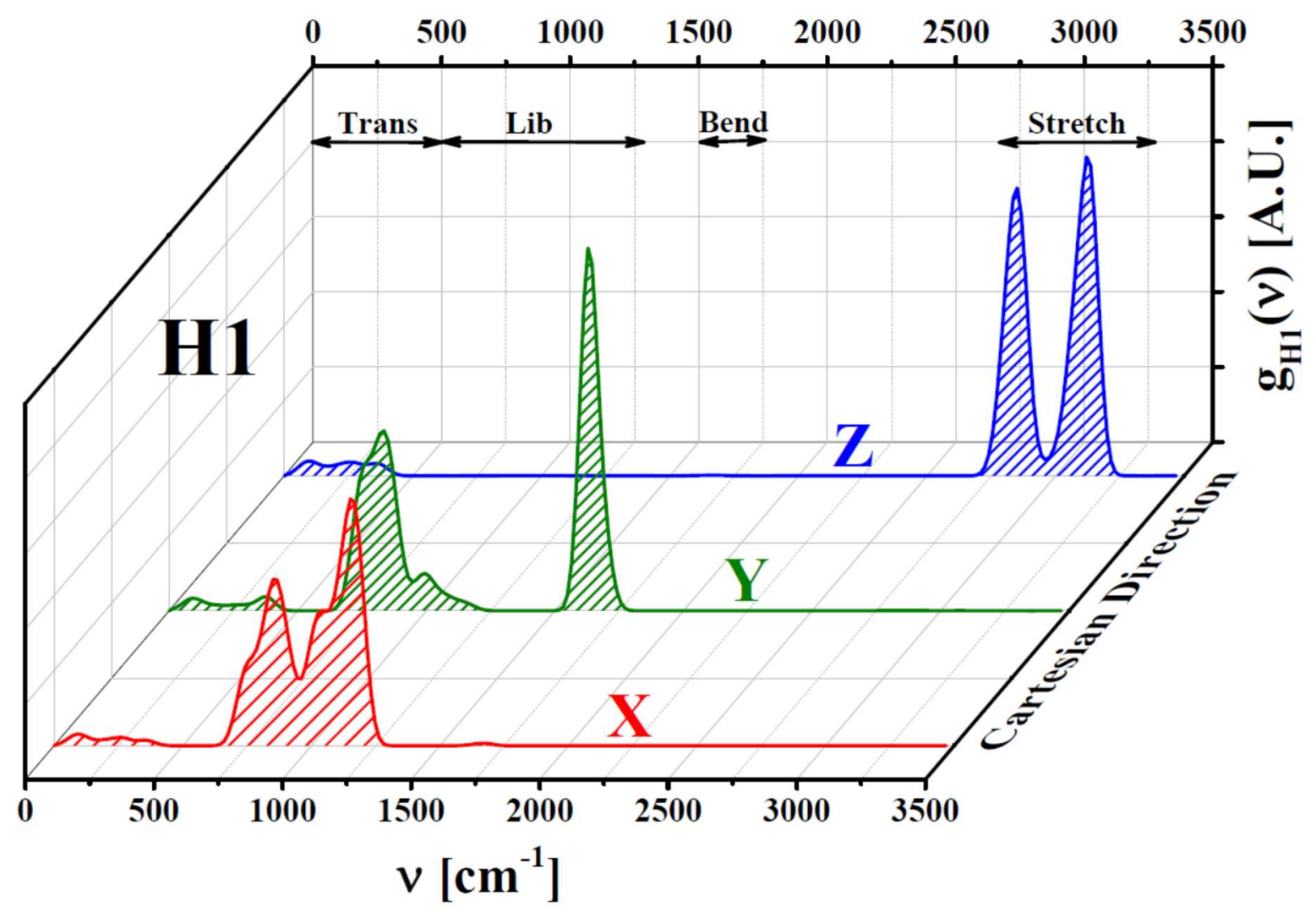

Fig. 3 Calculated Cartesian components (X -red, Y-green, Z-blue) of the H1-VDOS, $\mathrm{g}_{\mathrm{H} 1}(\mathrm{v})$. Assignments of phonon bands to $\mathrm{H}_{2} \mathrm{O}$ lattice (translation and libration) and internal (bending and stretching) are indicated.

Figs. 3 and 4 provide a clear view of the strong anisotropy of the protons VDOS in $\mathrm{H}_{2} \mathrm{O}$. Overall, the anisotropies emerging from those Figures fully comply with the relative orientations between the two $\mathrm{OH}$ bonds in the chosen $\mathrm{XYZ}$ axes system (Fig. 1). It may first 
be noted in Fig. 4 that while the translational phonon band $\left(0-500 \mathrm{~cm}^{-1}\right)$ of either proton along the three Cartesian directions are practically the same (nearly isotropic translational VDOS), all remaining bands, of libration (630-1330 $\left.\mathrm{cm}^{-1}\right)$, bend (1500-1750 $\left.\mathrm{cm}^{-1}\right)$ and stretch (2700$3500 \mathrm{~cm}^{-1}$ ), possess markedly different intensities along each direction in accord with the protons movements. Accounting for the stretch bands in Fig. 3 for example, $\mathrm{H}_{1}$ which vibrates along $\mathrm{OH}_{1}$ that is directed along $\mathrm{Z}$, possess high intensity phonons (solid blue lines in Fig. 3) compared to nearly zero intensities along the $\mathrm{X}$ and $\mathrm{Y}$ directions (solid red and green lines in Fig. 3 respectively).

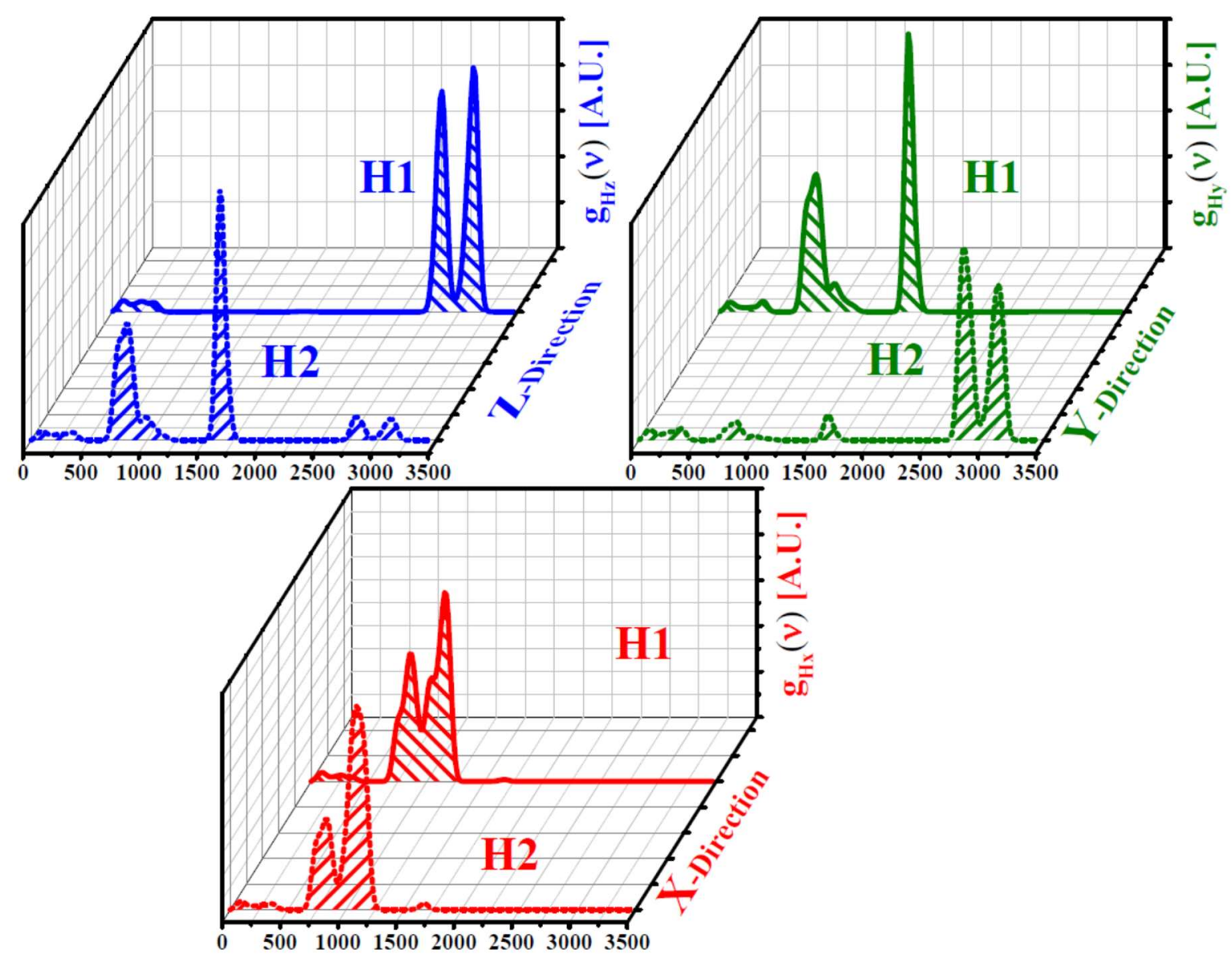

Fig. 4 Calculated Cartesian components (X -red, Y-green, Z-blue) of the H1- (solid) and H2(dotted) VDOS. Abscissa value are frequencies in $\mathrm{cm}^{-1}$ units.

As for the $\mathrm{H}_{2}$ atom whose $\mathrm{OH}_{2}$ bond is perpendicular to $\mathrm{X}$ and nearly normal to $\mathrm{Z}$ (aligned by $\sim 17^{\circ}$ with respect to $\mathrm{Y}$ ), it exhibits mostly a large Y VDOS component along the stretch 
band and a much smaller $\mathrm{Z}$ component (Fig. 4). Along the $\mathrm{X}$ direction, being normal to the $\mathrm{H}_{2} \mathrm{O}$ plane, both protons have negligible $\mathrm{X}$ stretch components (Fig. 4). It may be also expected that both protons have comparable librational and nearly zero bend intensities along $\mathrm{X}$, while along $\mathrm{Y}$ those of $\mathrm{H}_{2}$ are by far larger than those of $\mathrm{H}_{1}$. All those features may be easily understood by a consideration of Fig. 1.

The above VDOS calculation may also be validated by accounting for: (1) the total $\mathrm{H}_{2} \mathrm{O}-$ $\operatorname{VDOS} \sum_{l}\left[g_{H_{1 l}}(\mathrm{v})+g_{O_{l}}(\mathrm{v})+g_{H_{2 l}}(\mathrm{v})\right]$ from which the averages of the five fundamental frequencies (weighted by $g_{\mathrm{H}_{2} \mathrm{O}_{l}}(\mathrm{v})$ ) may be extracted, and (2) the averaged energy fractions shared by the proton, $\left[g_{H_{1 l}}(\mathrm{v})+g_{H 2_{l}}(\mathrm{v})\right] / 2$, in each fundamental energy band of the total $\mathrm{H}_{2} \mathrm{O}-$ VDOS. The results (summarized in Table I) may then by compared to the experimental frequencies of $\mathrm{H}_{2} \mathrm{O}$ and to the $\mathrm{SE}$ calculated energy fractions.

Table I Averaged frequencies $\left(v_{j}\right)$ of the five fundamental modes of motion of $\mathrm{H}_{2} \mathrm{O}$ and their corresponding kinetic energy fractions $\left(\mathrm{S}_{j}\right)$ shared by the protons. The calculated ( $p$ VDOS) frequencies and energy fractions are compared to experimental and SE results respectively.

\begin{tabular}{cc|cc}
\multicolumn{2}{c|}{} & \multicolumn{2}{|c}{$\mathrm{S}_{j}$} \\
$p$ VDOS & $\mathrm{v}_{j}\left[\mathrm{~cm}^{-1}\right]$ & $p$ VDOS & $\mathrm{SE}$ \\
\hline \hline 223 & 207 & 0.048 & 0.056 \\
950 & 782 & 0.481 & 0.475 \\
1641 & 1650 & 0.159 & 0.153 \\
2833 & 3085 & 0.157 & 0.154 \\
3101 & 3220 & 0.158 & 0.159
\end{tabular}

It may be seen from Table I that the calculated average frequencies differ by $10 \%$ at most while those of libration present a ca. $22 \%$ deviation. As for the energy fractions, they differ by $4 \%$ at most, with the only exception of the translational fractions which differ by $14 \%$. The above two large differences may be understood by accounting for the wide ranges of reported librational and translational frequencies in ice which easily cover the above two deviations; these facts support the validity of the above calculation.

The calculated Cartesian components of the H1-, H2- and O-VDOS were used as input data in Eq. 4 for deducing their KE components, $K_{l}(H, O)$. The results are given in Table II. 
Table II. Deduced $\mathrm{Ke}_{l}(\mathrm{~A})\left(l=\mathrm{x}, \mathrm{y}, \mathrm{z} ; \mathrm{A}=\mathrm{H}_{1}, \mathrm{O}, \mathrm{H}_{2}\right)$ values at 0 and $271 \mathrm{~K}$ in meV units. $\mathrm{Ke}_{t o t}(\mathrm{~A})=\sum_{l=x, y, z} K e_{l}(A)$ refer to the total (isotropic) $\mathrm{KE}$ value.

\begin{tabular}{c|c|c|c|c|c|c}
\multirow{2}{*}{$K_{\boldsymbol{K}}(\mathbf{A})$} & \multicolumn{5}{c}{$\mathrm{H}_{1} \mathrm{OH}_{2}$} \\
\cline { 2 - 7 } & \multicolumn{3}{|c|}{ ZPKE } & \multicolumn{3}{c}{$\mathbf{2 7 1} \mathbf{K}$} \\
\hline $\boldsymbol{l}$ & $\mathbf{H}_{1}$ & $\mathbf{H}_{2}$ & $\mathbf{O}$ & $\mathbf{H}_{1}$ & $\mathbf{H}_{2}$ & $\mathbf{O}$ \\
\hline $\mathbf{X}$ & 30.3 & 30.6 & 8.1 & 30.9 & 31.2 & 14.3 \\
\hline $\mathbf{Y}$ & 36.7 & 80.5 & 13.9 & 37.4 & 80.9 & 19.4 \\
\hline $\mathbf{Z}$ & 87.3 & 42.8 & 14.3 & 87.7 & 43.4 & 20.0 \\
\hline $\mathbf{K e}_{\text {tot }}(\mathbf{A})$ & 154 & 154 & 36.3 & 156 & 155.5 & 53.7
\end{tabular}

The KE values reveal the VDOS anisotropy. The first feature to be noted in Table I is that the total (isotropic) KE values of both $\mathrm{H}_{1}$ and $\mathrm{H}_{2}$ are practically the same $(\sim 155 \pm 1 \mathrm{meV})$, regardless of their differently oriented $\mathrm{OH}$ bonds. Nonetheless, their Cartesian components are clearly different. Note also that their ZPKE values are smaller by only $\sim 1 \%$ compared to the total values at $271 \mathrm{~K}$, a direct result of the huge ( $\sim 99 \%)$ zero point contribution to $\mathrm{Ke}(\mathrm{H})$. The calculated value of $\operatorname{Ke}(\mathrm{O})$ is also in very good agreement with experiment and its zero point part is also fairly large $(\sim 67 \%)$, however not as huge as that of the lighter proton.

By accounting for the five characteristic phonon bands of the $\mathrm{H}_{1}$-VDOS in ice (Fig. 3), the relative contributions of each Cartesian component of each mode of motion to $\operatorname{Ke}\left(\mathrm{H}_{1}\right)$ were deduced. The results are presented in Fig. 5 together with the total shares of each mode in $\mathrm{Ke}\left(\mathrm{H}_{1}\right)$. Fig. 5 provides a clear mapping of the vibrational interplay underlying $\mathrm{Ke}\left(\mathrm{H}_{1}\right)$; here again, the results are in full accordance with the chosen molecular orientation (Fig. 1): all translational components contribute equally and negligibly to $\mathrm{Ke}\left(\mathrm{H}_{1}\right)$. The stretching part of $\mathrm{Ke}\left(\mathrm{H}_{1}\right)$ is contributed only by the $\mathrm{Z}$ component of the $\mathrm{H}_{1}$-VDOS. The $\mathrm{H}_{2} \mathrm{O}$ bending part arise only from the $\mathrm{Y}$ component of the $\mathrm{H}_{1}$-VDOS (meaning that the internal modes only contribute to the $\mathrm{H}_{2} \mathrm{O}$ planar motion, the $\mathrm{X}$ component of $\mathrm{Ke}\left(\mathrm{H}_{1}\right)$ is contributed mainly by the out-of-plane libration of $\mathrm{H}_{2} \mathrm{O}$ around the $\mathrm{Y}$-axis. Finally, the libration of $\mathrm{H}_{2} \mathrm{O}$ around $\mathrm{Z}$ (the $\mathrm{OH}_{1}$ direction) has no contribution to $\mathrm{Ke}(\mathrm{H} 1)$. 


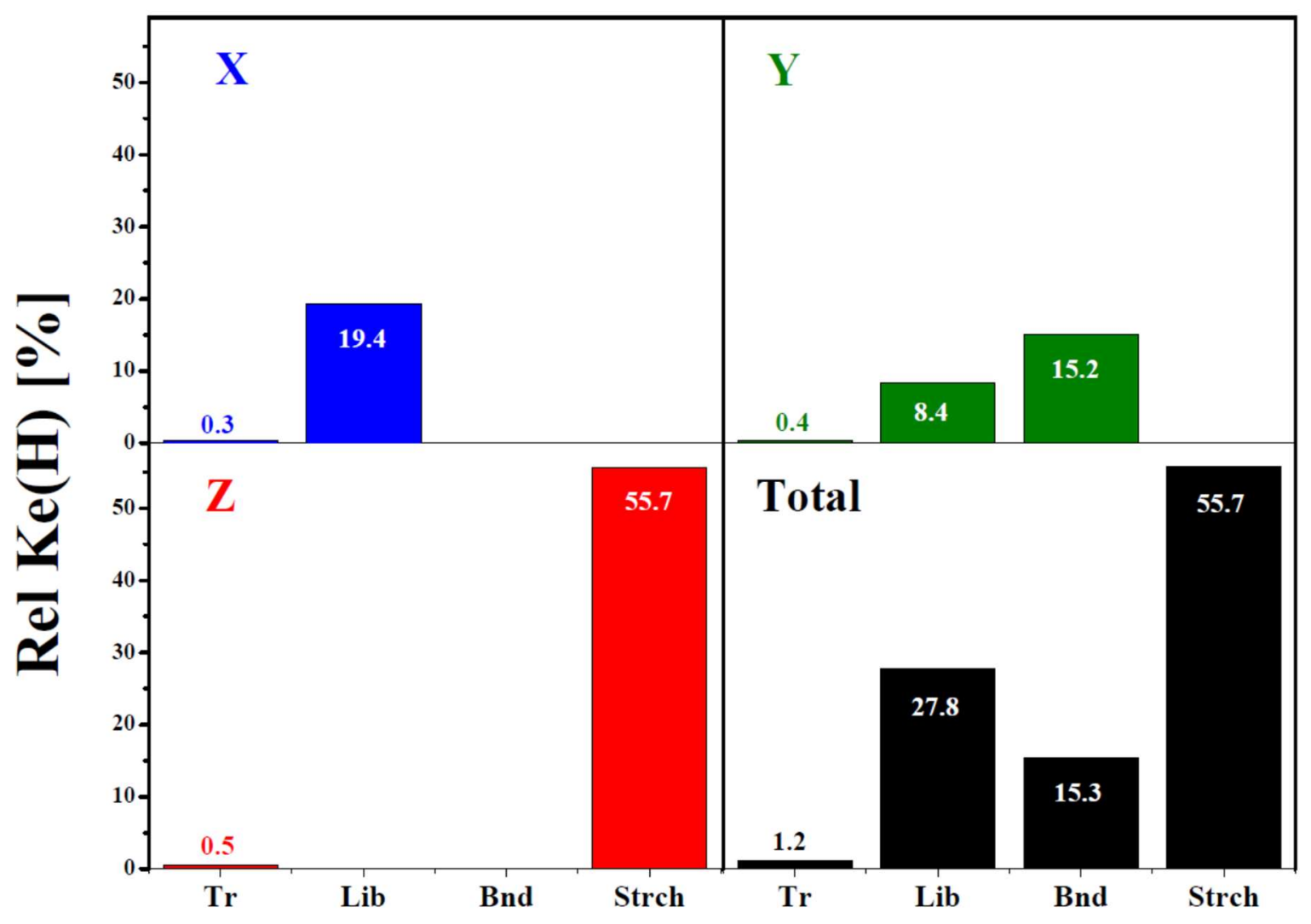

Fig. 5 Fractional fundamental parts of $\mathrm{Ke}(\mathrm{H} 1)$ and of its Cartesian components.

Such a rich informative mapping of the $\operatorname{Ke}(\mathrm{H})$ anisotropy could be highly valuable in exploring quantum competing effects such as those reported by DINS to occur upon melting of heavy water. ${ }^{5}$ It was reported in Ref. 5 that upon transition from the liquid to the solid, the DINS data show substantial increase in $\mathrm{Ke}_{\mathrm{x}}(\mathrm{D})$ (associated with motion normal to the $\mathrm{D}_{2} \mathrm{O}$ plane) accompanied with a corresponding large decrease in $\mathrm{Ke}_{\mathrm{z}}(\mathrm{D})$ (associated with motion parallel to the covalent $\mathrm{O}-\mathrm{D}$ bond). The opposite changes in those KE components, which lead to a much smaller change in the total kinetic energy, were viewed as a signature of CQEs in $\mathrm{Ke}(\mathrm{H})$. Here, the more hindered librations in the solid phase and the weakening of the covalent bond, are consistent with the red shift of the stretch peak observed in ice. A $p$ VDOS analysis of liquid and solid $\mathrm{D}_{2} \mathrm{O}$ can thus provide a direct detailed measure for the above effect. 


\section{Comparison with experiment}

It should first be noted that the two protons of $\mathrm{H}_{2} \mathrm{O}$ (Fig. 1) are distinguishable in terms of the relative orientation of their $\mathrm{OH}$ bonds with respect to the XYZ Cartesian directions. Note also that the molecular orientation in Fig. 1 was chosen so that the resulting KE components provide a direct comparison with experimental data. Moreover, in DINS experiments on isotropic samples, such as ice and liquid water, the neutron momentum distribution, $\mathrm{n}_{\mathrm{H}}(\mathrm{p})$, depends only on the proton momentum along the $\mathrm{q}$ direction. Here, the Cartesian components of $\mathrm{Ke}(\mathrm{H})$ are extracted from the measured neutron Compton profile, $\mathrm{NCP}$, by assuming that $\mathrm{n}_{\mathrm{H}}(\mathrm{p})$ arises from a spherical average of an anisotropic Gaussian distribution involving the three Cartesian variances of the total NCP. Differently from the above, in the $p$ VDOS approach, the Cartesian $\mathrm{Ke}(\mathrm{H})$ components are first calculated from which the mean value is then deduced.

Only two reports are available in the literature regarding the Cartesian components of the proton kinetic energy in light ice. These include $\mathrm{DINS}^{2,8}$ and $\mathrm{INS}^{2}$ studies, both treating $\mathrm{Ke}(\mathrm{H})$ along and perpendicular to the $\mathrm{OH}$ bond at $271 \mathrm{~K}$. The calculated and measured results are presented in Table III and Fig. 6.

Table III. Calculated and measured $\mathrm{Ke}(\mathrm{H})$ Cartesian components of ice Ih at $271 \mathrm{~K}$.

\begin{tabular}{c|c|cc|cc}
\multirow{2}{*}{$K_{i}\left(\mathrm{H}_{1}\right)$} & \multicolumn{3}{|c|}{ Calc. } & \multicolumn{2}{c}{ Exp. } \\
\cline { 2 - 6 } & DFT & \multicolumn{2}{|c}{ SE } & DINS $^{8}$ & DINS $^{2}$ \\
& H-VDOS & INS $^{2}$ & This study & & \\
\hline \hline $\mathbf{X}$ & 30.9 & $21.6 \pm 0.3$ & $23 \pm 3$ & $28 \pm 2$ & $29 \pm 2$ \\
$\mathbf{Y}$ & 37.4 & $34.4 \pm 1.2$ & $36 \pm 3$ & $38 \pm 5$ & $38 \pm 3$ \\
$\mathbf{Z}$ & 87.7 & $98.8 \pm 1.2$ & $93 \pm 3$ & $91 \pm 5$ & $87 \pm 3$ \\
\hline \hline $\mathbf{K e}_{\text {tot }}(\mathbf{A})$ & 156 & $154 \pm 2$ & $152 \pm 3$ & $157 \pm 2$ & $154 \pm 2$
\end{tabular}




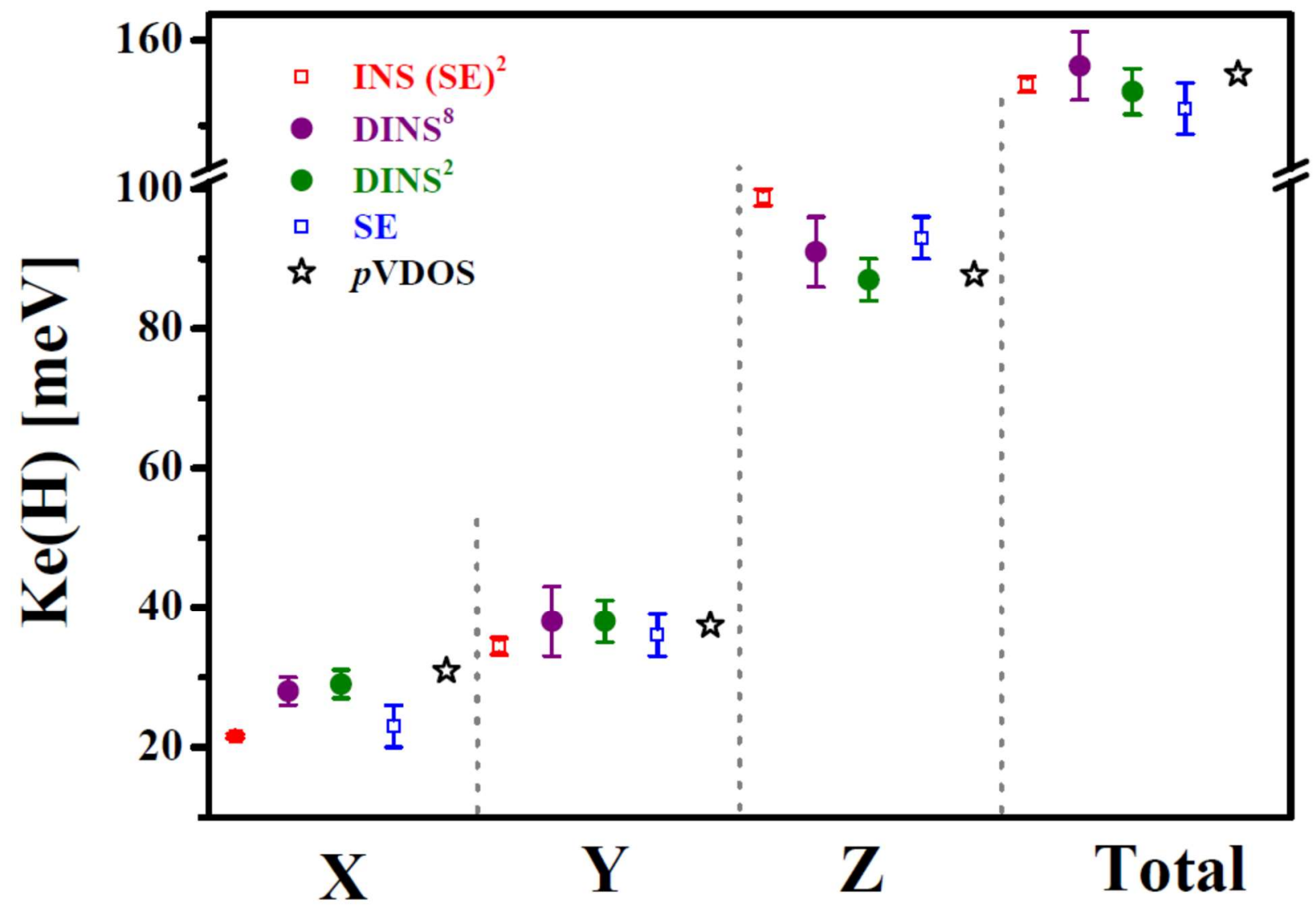

Fig. 6 Current SE and DFT- $p$ VDOS calculated values of the total $\mathrm{Ke}(\mathrm{H})$ and its Cartesian components versus published INS and DINS results.

Table III and Fig. 6 show that there is a very good agreement between the DINS measured values and the DFT calculation of the Cartesian components and also of the total $\operatorname{Ke}(\mathrm{H})$. However a relatively large deviation occurs from the SE results; this may be attributed to the uncertainties in the experimental translation and libration $\mathrm{H}_{2} \mathrm{O}$ frequencies used as input data. The above results emphasize the importance of the accuracy provided by the $p$ VDOS approach, because CQEs and NQEs that are considered as key effects underlying the behavior of $\mathrm{Ke}(\mathrm{H})$, can be very small and may thus be captured only by using the DFT$p$ VDOS calculations. Another important advantage of the $p$ VDOS approach is its great success in predicting the DINS $\mathrm{Ke}(\mathrm{H})$ value of nano-confined $\mathrm{H}_{2} \mathrm{O}$, e.g. in Beryl, while the SE approach entirely failed to do so. ${ }^{30}$ 


\section{Conclusions}

We report on the first DFT calculation of the Cartesian components of the partial H-VDOS in light ice, from which the components of the proton kinetic energy along and normal to the hydrogen bond were deduced. The results revealed a very good agreement with DINS measurements and were better than those obtained using the SE method. However, the two methods were both successful in treating pure $\mathrm{H}_{2} \mathrm{O}$ phases. Another advantage of applying the $p$ VDOS over SE is that it can predict the Cartesian components of $\mathrm{Ke}(\mathrm{H})$ thus providing a valuable tool for explaining signatures of CQEs and nuclear quantum effects (NQEs) in $\mathrm{H}_{2} \mathrm{O}$ phases and its vibrational dynamics. The main advantage of the $p$ VDOS over SE was revealed in deducing the $\mathrm{Ke}(\mathrm{H})$ value in nanoconfined water for which the SE approach was invalid.

\section{Acknowledgements}

FB and PV acknowledge the Research Council of Norway for providing the computer time (project number NN2875k) at the Norwegian supercomputer facility.

\section{References}

${ }^{1}$ Y. Finkelstein and R. Moreh, Chem. Phys. 431-432, 58 (2014).

${ }^{2}$ C. Andreani, G. Romanelli, and R. Senesi, Chem. Phys. 427, 106 (2013).

${ }^{3}$ Y. Finkelstein and R. Moreh, Curr. Phys. Chem. 7, 3 (2017).

4 a. Parmentier, J.J. Shephard, G. Romanelli, R. Senesi, C.G. Salzmann, and C. Andreani, J. Phys. Chem. Lett. 6, 2038 (2015).

${ }^{5}$ G. Romanelli, M. Ceriotti, D.E. Manolopoulos, C. Pantalei, R. Senesi, and C. Andreani, J. Phys. Chem. Lett 4, 3251 (2013).

${ }^{6}$ M. Ceriotti and D.E. Manolopoulos, Phys. Rev. Lett. 109, 100604 (2012).

${ }^{7}$ G. Romanelli, R. Senesi, X. Zhang, K.P. Loh, and C. Andreani, Phys. Chem. Chem. Phys. 17, 31680 (2015).

${ }^{8}$ C. Andreani, G. Romanelli, and R. Senesi, J. Phys. Chem. Lett. 7, 2216 (2016).

${ }^{9}$ G. Romanelli, F. Fernandez-Alonso, and C. Andreani, J. Phys. Conf. Ser. 571, 012003 (2014).

${ }^{10}$ R. Moreh and D. Nemirovsky, J. Chem. Phys. 131, 054305 (2009).

${ }^{11}$ R. Moreh and D. Nemirovsky, J. Chem. Phys. 130, 174303 (2009). 
${ }^{12}$ R. Moreh and Y. Finkelstein, Phys. Rev. B 61, 7700 (2000).

${ }^{13}$ R. Moreh, Y. Finkelstein, and H. Shechter, Phys. Rev. B 53, 16006 (1996).

${ }^{14}$ R. Moreh, H. Pinto, Y. Finkelstein, V. Volterra, Y. Birenbaum, and F. Beguin, Phys. Rev. B 52, 5330 (1995).

${ }^{15}$ Y. Finkelstein, O. Beck, R. Moreh, D. Jäger, U. Kneissl, J. Margraf, H. Maser, and H.H. Pitz, Phys. Rev. B 58, 4166 (1998).

${ }^{16}$ R. Moreh, O. Beck, D. Jäger, Y. Finkelstein, U. Kneissl, J. Margraf, H. Maser, and H. Pitz, Phys. Rev. B 56, 187 (1997).

${ }^{17}$ O. Beck, Y. Ruf, Y. Finkelstein, M. Cardona, T.R. Anthony, D. Belic, T. Ecket, D. Jager, U. Kneissl, H. Masser, R. Moreh, A. Nord, H.H. Pitz, and A. Wolpert, J. Appl. Phys. 83, 5484 (1998).

${ }^{18}$ Y. Finkelstein, R. Moreh, S.L. Shang, Y. Shchur, Y. Wang, and Z.K. Liu, J. Chem. Phys. 144, 054302 (2016).

${ }^{19}$ R. Senesi, A. Pietropaolo, A. Bocedi, S. Pagnotta, and F. Bruni, Phys. Rev. Lett. 98, 138102 (2007).

${ }^{20}$ A. Pietropaolo, D. Fernandez-Cañoto, E. Perelli-Cippo, S. Dirè, and P. Prosposito, Phys. Rev. B - Condens. Matter Mater. Phys. 77, 014202 (2008).

${ }^{21}$ V. Garbuio, C. Andreani, S. Imberti, A. Pietropaolo, G.F. Reiter, R. Senesi, and M.A. Ricci, J. Chem. Phys. 127, 154501 (2007).

${ }^{22}$ G.F. Reiter, A.I. Kolesnikov, S.J. Paddison, P.M. Platzman, A.P. Moravsky, M.A. Adams, and J. Mayers, Phys. Rev. B 85, 045403 (2012).

${ }^{23}$ A.I. Kolesnikov, G.F. Reiter, N. Choudhury, T.R. Prisk, E. Mamontov, A. Podlesnyak, G. Ehlers, A.G. Seel, D.J. Wesolowski, and L.M. Anovitz, Phys. Rev. Lett. 116, 167802 (2016).

${ }^{24}$ J. a Morrone, V. Srinivasan, D. Sebastiani, and R. Car, J. Chem. Phys. 126, 234504 (2007).

${ }^{25}$ C.P. Herrero and R. Ramirez, Chem. Phys. 461, 125 (2015).

${ }^{26}$ C.P. Herrero and R. Ramirez, J. Chem. Phys. 137, 104505 (2012).

${ }^{27}$ R. Ramirez and C.P. Herrero, Phys. Rev. B - Condens. Matter Mater. Phys. 84, 064130 (2011).

28 a. Parmentier, J.J. Shephard, G. Romanelli, R. Senesi, C.G. Salzmann, and C. Andreani, J. Phys. Chem. Lett. 6, 2038 (2015).

${ }^{29}$ A. Striolo, A.A. Chialvo, K.E. Gubbins, and P.T. Cummings, J. Chem. Phys. 122, $234712(2005)$.

${ }^{30}$ Y. Finkelstein, R. Moreh, S.L. Shang, Y. Wang, and Z.K. Liu, J. Chem. Phys. 146, 124307 (2017).

${ }^{31}$ C. Andreani, M. Krzystyniak, G. Romanelli, R. Senesi, and F. Fernandez-alonso, Adv. Phys. 65, 1 (2017). 
${ }^{32}$ Y. Finkelstein and R. Moreh, J. Chem. Phys. 139, 044716 (2013).

${ }^{33}$ G. Kresse and J. Furthmiiller, Comput. Mater. Sci. 6, 15 (1996).

${ }^{34}$ G. Kresse and J. Furthmu, Phys. Rev. B 54, 11169 (1996).

35 J.P. Perdew, K. Burke, and M. Ernzerhof, Phys. Rev. Lett. 77, 3865 (1996).

${ }^{36}$ A. Togo and I. Tanaka, Scr. Mater. 108, 1 (2015).

${ }^{37}$ H.J. Monkhorst and J.D. Pack, Phys. Rev. B 13, 5188 (1976).

${ }^{38}$ T. Ikeda-fukazawa, S. Horikawa, T. Hondoh, and K. Kawamura, J. Chem. Phys. 117, 3886 (2002).

${ }^{39} \mathrm{Https}$ //atztogo.github.io/phonopy/setting-tags.html\#projection-direction-tag 